\title{
Breaking Barriers Amid the Pandemic: The Status of Telehealth in Southeast Asia and its Potential as a Mode of Healthcare Delivery in the Philippines
}

Aitana D. Macariola ${ }^{1}$, Theara Mae C. Santarin ${ }^{1}$, Ferianne J oy M. Villaflor ${ }^{1}$, Leofe Marie G. Villaluna ${ }^{1}$, Rea Shane L. Yonzon ${ }^{1}$, J amie L. Fermin ${ }^{2}{ }^{3}$, Shaira L. Kee ${ }^{1,3}$, Nouar AlDahoul ${ }^{4,3}$, Hezerul A. Karim ${ }^{4 *}$, Myles J oshua T. $\operatorname{Tan}^{5,3,1^{*}}$

${ }^{1}$ Department of Natural Sciences, University of St. La Salle, Philippines, ${ }^{2}$ Department of Electronics Engineering, University of St. La Salle, Philippines, ${ }^{3}$ Yo-Vivo Corporation, Philippines, ${ }^{4}$ Faculty of Engineering, Multimedia University, Malaysia, ${ }^{5}$ Department of Chemical Engineering, University of St. La Salle, Philippines

Submitted to J ournal:

Frontiers in Bioengineering and Biotechnology

Specialty Section:

Bionics and Biomimetics

Article type:

Opinion Article

Manuscript ID:

754011

Received on:

05 Aug 2021

Journal website link:

www. frontiersin.org 


\section{Conflict of interest statement}

\section{The authors declare a potential conflict of interest and state it below}

J.L.F., S.L.K., N.A., and M.J.T.T. are affiliated with Yo-Vivo Corporation, a telemedicine service provider based in the Philippines. Yo-Vivo Corporation did not financially support this work.

\section{Author contribution statement}

Conceived the work: A.D.M., T.M.C.S., F.J.M.V., L.M.G.V., R.S.L.Y., J.L.F., S.L.K., N.A., H.A.K., and M.J .T.T.; Drafted the article: A.D.M., T.M.C.S., F.J .M.V., L.M.G.V., and R.S.L.Y.; Critically revised the manuscript: J.L.F., S.L.K., N.A., H.A.K., and M.J .T.T.; Acquired funding: H.A.K. All authors contributed to the article and approved the submitted version.

\section{Keywords}

telehealth, Telemedicine, Philippines, southeast asia, COVID-19, pandemic, Industry - 4.0, medicine 4.0

\section{Contribution to the field}

From a public health perspective, this opinion article discusses the necessity to push for telehealth in the Philippines as a mode of healthcare delivery, based on lessons from other Southeast Asian countries. With the recent pandemic, the Philippines has witnessed the potential of telehealth to cater to the healthcare needs of the public. Telehealth fills in the gaps brought about by the pandemic, delivering quality healthcare services to Filipinos. We hope that this encourages both the public and private sectors to lend their full support to efforts to promote telehealth, particularly in the Philippines.

\section{Funding statement}

The publication of this article was funded by Multimedia University, Malaysia. 


\section{Breaking Barriers Amid the Pandemic: The Status of}

Telehealth in Southeast Asia and its Potential as a Mode of Healthcare Delivery in the Philippines

Aitana Dy Macariola ${ }^{1 \dagger}$, Theara Mae Capacion Santarin ${ }^{1 \dagger}$, Ferianne Joy Manday Villaflor ${ }^{1 \dagger}$, Leofe Marie Guintos Villaluna ${ }^{1 \dagger}$, Rea Shane Leonora Yonzon $^{1 \dagger}$, Jamie Ledesma Fermin ${ }^{2,3}$,Shaira Limson Kee ${ }^{1,2}$, Nouar AlDahoul $^{2,4}$, Hezerul Abdul Karim ${ }^{4 *}$, Myles Joshua Toledo Tan ${ }^{1,2,5 *}$

†These authors have contributed equally to this work and share first authorship.

${ }^{1}$ Department of Natural Sciences, University of St. La Salle, Bacolod, Philippines

${ }^{2}$ Yo-Vivo Corporation, Bacolod City, Philippines

${ }^{3}$ Department of Electronics Engineering, University of St. La Salle, Bacolod, Philippines

${ }^{4}$ Faculty of Engineering, Multimedia University, Cyberjaya, Malaysia

${ }^{5}$ Department of Chemical Engineering, University of St. La Salle, Bacolod, Philippines

* Correspondence:

Hezerul Abdul Karim

hezerul@mmu.edu.my

Myles Joshua Toledo Tan

mj.tan@usls.edu.ph

Keywords: telehealth, telemedicine, Philippines, Southeast asia, industry 4.0, medicine 4.0, covid-19, pandemic 


\section{Introduction}

Southeast Asia (SEA) is a geographical bloc that is sociologically, politically, and economically diverse. This diversity has led to heterogeneity in levels of development of healthcare systems in its member states (Chongsuvivatwong et al., 2011). Nevertheless, countries in SEA share joint commitments, particularly in achieving Universal Health Coverage. Their efforts toward this goal has progressed significantly, as evidenced by the increasing availability of preventive and curative care services across SEA (Van Minh et al., 2014).

As it is, the healthcare system of SEA struggles to meet the ever-changing demands of its aging population that has become more prosperous and aware of its human rights (Chongsuvivatwong et al., 2011). The problem is the insufficient healthcare workforce of SEA, with only 1.93 doctors per 10 ooo population in Cambodia as the lowest, and 22.94 doctors per 10000 population in Singapore as the highest, according to the World Health Organization (WHO). In the report, eight out of 11 SEA countries (Cambodia, Indonesia, Laos, Myanmar, Philippines, Thailand, Timor-Leste, and Vietnam) have doctor-population ratios lower than the WHO recommendation of 10 doctors per 10 ooo population. This translates to immense amounts of work for doctors serving patients in the region.

Excessive workload leads to physician burnout, impairing their efficiency and increasing medical errors (Michtalik et al., 2013). Moreover, healthcare facilities, particularly public hospitals, are frequently overcrowded in low-resourced SEA countries. The situation is due to a shortage of beds, staff, supplies, and diagnostic equipment (Lim et al., 2014), which causes delays in care, and leads to many patients leaving without being examined. Since most individuals in urban areas have hectic schedules, many avoid obtaining medical treatment entirely (Taber et al., 2015). This medical avoidance can lead to delays in diagnosis and treatment, resulting in higher morbidity and mortality in various cases (Moser et al., 2006).

Moreover, health workers in SEA are primarily concentrated in urban areas because of greater prospects and pay in these places, leaving rural areas understaffed (Kanchanachitra et al., 2011; Nair \& Webster, 2013). This compels rural folk to seek medical treatment in cities when medical specialists are not accessible in their locality. This problem is often accompanied by distant and lengthy travel, additional expenses, and a lack of reliable transportation (Douthit et al., 2015).

Healthcare-associated infections with a pooled prevalence of $9.0 \%$ also pose a challenge in patient care throughout SEA (Ling et al., 2015). When visiting healthcare facilities, an individual is exposed to pathogens from various sources. For instance, if an individual 
infected with measles is also present in the waiting room, the probability of transmission is more than $10 \%$ on $59 \%$ of occasions (Beggs et al., 2010). Children, in particular, have an increased risk of direct infections from touching toys and playing closely with other sick children in waiting rooms (Merriman et al., 2002). As a result, some individuals decide not to seek medical attention to avoid being around ill individuals.

With progress in technology over the past decades, access to services have indeed improved, especially in the healthcare and communication industries. Digital health drivers brought by Industry 4.0 have immensely enhanced healthcare services (Ye, 2020; Ting et al., 2020), making them faster and more efficient globally. In fact, in technologically advanced countries, healthcare has started shifting toward remote services, making it easier to receive medical care (Kruse et al., 2017). This, consequently, has given rise to the telehealth industry.

Telehealth is integrated into the system to make healthcare more accessible and affordable. In the United States (US), the number of annual telehealth visits increased from 206 in 2005 to 202,314 in 2017 (Barnett et al., 2018). Furthermore, the outbreak of the COVID-19 pandemic in 2020 also caused an acceleration in telehealth usage. In fact, the increase in telehealth usage coincided with the rise in COVID-19 cases (Weber et al., 2020). Compared to the same month in 2019, US data showed a $154 \%$ increase in telehealth usage in March 2020 (Koonin et al., 2020). It seems as though the pandemic acted as a catalyst for the modernization of healthcare.

Telehealth has allowed doctors to provide health services remotely (Dorsey \& Topol, 2016). Its advantages are not only concentrated on patient wellbeing but also on that of healthcare workers. It makes use of health information technology (HIT) and remote patient monitoring (RPM) to deliver consultations at a distance (Ye, 2020). With the help of advancements in the internet of things (IoT), biomedical sensor technologies, and modern communication, patient monitoring at home has become possible (Ahmad et al., 2021; Malasinghe et al., 2019). These medical devices, which ease the workloads of healthcare workers, are innovations brought about by biomedical engineers. Biomedical engineering (BME) is a discipline that uses engineering principles in medicine and biology to produce advanced medical technologies that improve healthcare systems (Al Asif et al., 2018). Biomedical engineers are essential members of the health workforce in countries with well-established healthcare systems. In fact, according to a study by Fermin \& Tan (2020), healthcare improvements can be observed along with an increase in BME research in ASEAN countries. As seen from the same study, the products of this field, which include telehealth, are fundamental drivers for an improved healthcare system. 
The rapid advancements in medical technologies reinforce the already expanding demand for Telehealth globally (Swanepoel et al., 2010). In 2017, the WHO reported that there would be a significant increase in healthcare expenses in Asia in the next ten years (Raghavan et al., 2021). Projections predict a 1.2\% annual growth per capita in health expenditures in Brunei, and 7.4\% growth in Myanmar from 2014 to 2040 (Dieleman et al., 2017). This increase is mainly driven by population growth, indicating that healthcare services will also grow in demand. Raghavan et al. (2021) predicted that by 2050, the escalating population in Asia would increase the demand for healthcare facilities. With this significant growth in the population, telehealth could become a viable solution for these problems in the future.

The telehealth industry is bound to become the solution to the aforementioned crises. Most SEA countries have varying telehealth guidelines that primarily focus on ethical and clinical aspects but fail to discuss the technology needed to deliver these services (Sabrina \& Defi, 2021). Thus, detailed technology guidelines should be established. These guidelines will enable countries to have a basis for implementing telehealth and to ensure high-quality care. Government agencies should also implement necessary measures to fortify the telehealth industry and fill the overwhelming healthcare gap.

\section{The Technological Drivers of Telehealth}

Telehealth is the result of the entry of advanced technology into the health industry. With the upgrade and fusion of these technologies, the potential of telehealth as an ecosystem within the larger global healthcare sphere is eminent.

First, AI is utilized in electronic health records, health monitoring, and many more. Computers in clinical imaging can assist doctors in the interpretation of medical images. It is commonly used in Radiology and has increased the accuracy, efficiency, and productivity in the specialty (Jalal et al., 2019).

Jagadeeswari et al. (2018) recently reviewed the emerging technologies in a personalized healthcare system, including IoT, cloud computing, big data analytics, and mobile technology. The combination of these technologies has made monitoring easier for both the caregiver and the patient. For instance, mobile applications can show the user's current health condition and the system can also alert healthcare providers when emergency medical services are needed.

The rise of the $5 \mathrm{G}$ mobile network can deliver higher data speeds, lower network latency, and a greater capacity for users. In this case, $5 \mathrm{G}$ will benefit both the patient and the telehealth provider by making communication over a distance devoid of delay (Siriwardhana et al., 2020). 
Through augmented reality (AR), users can experience the physical world with added digital elements like sounds or visuals. Virtual reality (VR), on the other hand, provides users with $3 \mathrm{D}$ views of artificial environments. Although AR/VR in medicine is not widely adopted, it is well-reviewed by several studies (Eckert et al., 2019). Thus, it can serve as an alternative to medical training and treatment in physical settings.

$3 \mathrm{D}$ printing or additive manufacturing (AM) creates $3 \mathrm{D}$ objects based on digital models. During the COVID-19 pandemic, there was an increased demand for protective equipment. AM was used as a supplementary manufacturing process to address emergent supply needs (Tareq et al., 2021). Moreover, digital designs of medical supplies are made available to the public by the National Institutes of Health (NIH) $3 \mathrm{D}$ Print Exchange. This online portal by the NIH could promote the use of AM in the medical industry.

We are in the middle of a fourth technological revolution in medicine - Medicine 4.o. These technologies are only some of the key factors driving the transition. Medicine 4.0 will combine current and evolving technologies to create a more efficient healthcare system. Along with this revolution in medicine is the transition into Society 5.0. This society will be one that fully uses digital technology to resolve societal problems and achieve economic development. Simply put, technology won't only reshape the medical industry, but more importantly, all of society.

\section{The Status of Telehealth in Southeast Asia}

Some SEA countries have turned to telehealth to expand the scope of their healthcare systems. For instance, mobile applications have allowed users to report their health conditions and educate themselves about COVID-19. A real-time information system also helped hospitals in Chiang Mai by integrating their data on one platform, making patient monitoring and hospital referrals easier (Intawong et al., 2021). Thailand has also developed their eHealth Strategy 2017-2026 to employ digital technologies in the health sector effectively. According to the Thailand Ministry of Public Health (2017), central organizations for eHealth management will be established to ensure trust and protection for the health industry and its consumers.

Telehealth has also yielded positive outcomes, especially during the pandemic in Singapore. For instance, medical personnel who have used the mobile messaging platform MyDoc $\AA$, strongly agreed that the application should replace current peer-to-peer communication systems in an orthopedic clinic (Daruwalla et al., 2014). Video conferences, mobile apps, and other telehealth platforms have also been found to be practical communication methods for mental health services like counseling and psychoeducation (Zhou et al., 2020). Moreover, the Singapore's Healthcare Services Act 
(HCSA) to be implemented in 2022 will replace the Private Hospitals and Medical Clinic Act (PHMCA) of 1980. In this act, the Singapore Ministry of Health (2021) will require a license for telehealth providers to ensure that they comply with safe practice guidelines.

In the Philippines, increased access to information and communication technologies over the years have led to the rise of telehealth. Because of the pandemic, teleconsultations can now be done through COVID-19 hotlines, websites, and mobile apps launched by several agencies and businesses. These include the Department of Health (DOH), Medgate, KonsultaMD, Medifi, HealthNow, AIDE, DOCPH, Yo-Vivo Health, and Lifeline. Moreover, the House of Representatives filed House Bill No. 7422, or the Philippine E-Health and Telemedicine Development Act (2020), which seeks to establish and develop Philippine telehealth industries using information and communications technologies (ICT) for the delivery of health services. However, this bill is still pending with the Committee on Health. Even before the pandemic, Fernandez-Marcelo et al. (2012) argued that certain factors need to be discussed to develop the telehealth industry. These factors include policies, capability building, and the collaboration of different sectors. Meanwhile, SEA countries like Indonesia, Malaysia, Singapore, Thailand, and Vietnam have already developed guidelines on telehealth (Intan Sabrina \& Defi, 2021).

\section{The Role of Telehealth in the COVID-19 Pandemic}

During the COVID-19 pandemic, healthcare systems were forced to adapt to remote delivery in order to minimize transmission of the virus. The need to accommodate both COVID-19 and COVID-19-free patients has also been a challenge. Many studies suggest that telehealth has improved healthcare provision during the COVID-19 pandemic and recommend it for public safety (Monaghesh \& Hajizadeh, 2020; Nguyen et al., 2008; Somsiri et al., 2020; Bagayoko et al., 2014). These studies also discuss how telehealth can contain the pandemic and preserve personal protective equipment. Telehealth has also improved the management of chronic diseases and has proven to save travel time and money (Somsiri et al., 2020; Xu et al., 2018). According to the Philippine Statistics Authority, the health expenditure on senior citizens has reached around 3.43 billion USD in 2020, of which 2.03 billion USD were out-of-pocket expenses while 1.4 billion USD were financed by Philippine Health Insurance Corporation, domestic revenue-based schemes, and other financing sources like those by health maintenance organizations. ${ }^{1}$ By investing in telehealth, nations can better healthcare provision, improve labor productivity and ultimately increase economic performance (Raghupathi \& Raghupathi, 2020). The ultimate advantages that telehealth brings to the public are convenience, security, and a safer healthcare alternative during the pandemic. 


\section{Barriers to Telehealth and Some Potential Solutions}

Although telehealth offers numerous benefits, some barriers require serious attention for telehealth to succeed. Based on the WHO, lack of policy in SEA is the main barrier to telehealth implementation. In the Philippines, for example, there are no existing legal frameworks for the implementation of telehealth. However, different eHealth initiatives have shown promise in breaking this barrier. The DOH has also proposed the Philippine eHealth Strategic Framework and Plan 2014-2020, which includes establishing telehealth infrastructures. On the legislative side, House Bills 7422 and 7153, which support the establishment of ICT and medical bases for eHealth services and solutions, have also been introduced. Furthermore, PhilHealth and its partners will finance applicable eHealth services, as per the National eHealth Steering Committee.

According to the Centers for Disease Control and Prevention (2020), poor internet connectivity, low cellular reception, technological illiteracy, and lack of access to gadgets are barriers to telehealth. Many Philippine schools lack computers, internet access, and trained ICT educators to increase digital literacy according to the Department of Information and Communication Technology. In Cambodia, only $32.4 \%$ of the population with tertiary education uses computers (Nit et al., 2021). Physicians who utilize virtual media in delivering healthcare services need formal training in the use of these media to guarantee outcomes similar to in-person consultations (Nochomovitz \& Sharma, 2017). As a solution, telehealth must be included in medical curricula for student-physicians to acquire the necessary education and training. According to Balaji and Clever (2021), it is essential to include telehealth exposure in different medical programs. Moreover, before residency, exposure of medical students to telehealth systems is critical. Proper education on modern ICT tools for older patients is also essential to address the challenges in the use of modern technologies (Holderried et al., 2021).

Internet speeds affect the quality of communication between patients and healthcare providers. The average internet speed in the Philippines is at $2.8 \mathrm{Mbps}$ (20.35USD/Mbps), while Indonesia's is at 2.2 Mbps (18.83USD/Mbps), and Malaysia's is at 4.3 Mbps (10.29USD/Mbps) (Salac \& Kim, 2016). Several studies report that the quality of home online health consultation systems are interrupted by slow internet speeds that result in poor audio and video quality, connection loss, and patients displeasure (Bernocchi et al., 2016; Dimitropoulos et al., 2017; Eslami Jahromi \& Ahmadian, 2018; Almathami et al., 2020). Hence, strengthening internet connectivity and cell reception, educating telecommunication users, and increasing access to gadgets are essential for the implementation of telehealth. 


\section{Discussion}

Telehealth is a healthcare revolution. It is not only driven by internet connectivity, but by several other technologies, as well. Though it has been long introduced in the Philippines, its potential hasn't been fully realized until the pandemic struck. The COVID-19 pandemic has pushed its development in the country further, making it the 'new normal' means of delivering healthcare. Unfortunately, telehealth training programs are limited, so distant and secluded communities remain uninformed. Hence, we suggest that local government units participate in the conduct of telehealth training for healthcare providers and information drives for the public. Educational institutions, including medical schools, in the country should also implement courses on both the provision and the use of telehealth into their curricula, to ensure that future healthcare providers and patients have the proper education and training to provide and receive medical care using available ICT tools.

The lack of access to technology necessary to obtain telehealth services also hinders successful telehealth implementation. For patients with limited access to necessary devices, same-day teleconsultations at local clinics may guarantee that all patients receive the medical care they need and deserve. Telehealth services must also be simple and straightforward for patients of all ages to utilize. Finally, we must remember that every community telehealth program is unique, and there is no one-size-fits-all approach to its implementation. Implementation strategies will differ from one healthcare environment to another due to numerous variables.

The establishment of telehealth as an alternative to traditional face-to-face healthcare services can improve the quality of life of many Filipinos. For this reason, the Philippine government must put greater emphasis on its implementation in the country, especially during this time of the pandemic. Collaboration with the government can expedite the implementation process and fill in existing gaps. Overall, it is possible to establish telehealth in the country despite the existing challenges. It is time to improve the country's healthcare system and give Filipinos equitable access to healthcare. Things might take time, but with proper planning, cooperation, and dedication in keeping everything running, good-quality care may just be beneath our fingertips. 


\section{Conflict of Interest}

J.L.F., S.L.K., N.A., and M.J.T.T. are affiliated with Yo-Vivo Corporation, a telemedicine service provider based in the Philippines. Yo-Vivo Corporation did not financially support this work.

\section{Author Contributions}

Conceived the work: A.D.M., T.M.C.S., F.J.M.V., L.M.G.V., R.S.L.Y., J.L.F., S.L.K., N.A., H.A.K., and M.J.T.T.; Drafted the article: A.D.M., T.M.C.S., F.J.M.V., L.M.G.V., and R.S.L.Y.; Critically revised the manuscript: J.L.F., S.L.K., N.A., H.A.K., and M.J.T.T.; Acquired funding: H.A.K. All authors contributed to the article and approved the submitted version.

\section{Funding}

The publication of this article was funded by Multimedia University, Malaysia.

\section{Contribution to the field}

From a public health perspective, this opinion article discusses the necessity to push for telehealth in the Philippines as a mode of healthcare delivery, based on lessons from other Southeast Asian countries. With the recent pandemic, the Philippines has witnessed the potential of telehealth to cater to the healthcare needs of the public. Telehealth fills in the gaps brought about by the pandemic, delivering quality healthcare services to Filipinos. We hope that this encourages both the public and private sectors to lend their full support to efforts to promote telehealth, particularly in the Philippines.

\section{References}

Ahmad, R. W., Salah, K., Jayaraman, R., Yaqoob, I., Ellahham, S., and Omar, M. (2021). The role of blockchain technology in telehealth and telemedicine. International Journal of Medical Informatics, 104399. doi: 10.1016/j.ijmedinf.2021.104399

Almathami, H. K. Y., Win, K. T., and Vlahu-Gjorgievska, E. (2020). Barriers and facilitators that influence telemedicine-based, real-time, online consultation at patients' homes: systematic literature review. Journal of Medicine Internet Research, 22 (2). doi: 10.2196/16407

Al Asif, M., Roy, S., Abdullah, A., Raihan, M., Akter, R., and Hossain, M. (2018). Role and impact of biomedical engineering discipline for developing country perspective. International Journal of Innovative Research in Computer Science \& Technology (IJIRCST) Volume-6, Issue-4. doi:10.21276/ijircst.2018.6.4.9

Balaji, A. and Clever, S. L. (2021). Incorporating medical students into primary care telehealth visits: tutorial. JMIR Med Educ, 7(2): e24300. doi:10.2196/24300 
Barnett, M. L., Ray, K. N., Souza, J., and Mehrotra, A. (2018). Trends in telemedicine use in a large commercially insured population, 2005-2017. Jama, 32O(20), 2147-2149.

doi:10.1001/jama.2018.12354

Beggs, C. B., Shepherd, S. J., and Kerr, K. G. (2010). Potential for airborne transmission of infection in the waiting areas of healthcare premises: Stochastic analysis using a Monte Carlo model. BMC Infect Dis. 10: 247. doi:10.1186/1471-2334-10-247

Bernocchi P, Vanoglio F, Baratti D, Morini R, Rocchi S, Luisa A, and Scalvini S. (2016). Home-based telesurveillance and rehabilitation after stroke: A real-life study. Top Stroke Rehabil. 23(2):106-115. doi: 10.1080/10749357.2015.1120453.

Centers for Disease Control and Prevention. (2020). Using Telehealth to Expand Access to Essential Health Services during the COVID-19 Pandemic. Centers for Disease Control and Prevention. https://www.cdc.gov/coronavirus/2019-ncov/hcp/telehealth.html. [Accessed July 17, 2021].

Chongsuvivatwong, V., Phua, K. H., Yap, M. T., Pocock, N., Hashim, J. H., and Chhem, R., et al. (2011). Health and health-care systems in Southeast Asia: Diversity and transitions. The Lancet. 377:9763, 429-437. doi:10.1016/So140-6736(10)61507-3

Daruwalla, Z. J., Wong, K. L., and Thambiah, J. (2014). The application of telemedicine in orthopedic surgery in Singapore: a pilot study on a secure, mobile telehealth application and messaging platform. JMIR mHealth and uHealth, 2(2), e3303. doi:10.2196/mhealth.3303

Department of Health (2021). Philippine eHealth strategic plan (PeHSP) 2014-2020. http://ehealth.doh.gov.ph/index.php/pehsp/overview [Accessed July 1, 2021].

Department of Information and Communication Technology. (2014). Philippine Digital Strategy. Department of Information and Communication Technology. https://dict.gov.ph/philippine-digital-strategy/. [Accessed July 12, 2021]

Dieleman, J. L., Campbell, M., Chapin, A., Eldrenkamp, E., Fan, V. Y., and Haakenstad, A., et al. (2017). Future and potential spending on health 2015-40: development assistance for health, and government, prepaid private, and out-of-pocket health spending in 184 countries. The Lancet, 389(10083), 2005-2030. doi:10.1016/So140-6736(17)30873-5

Dimitropoulos A, Zyga O, and Russ S. (2017). Evaluating the feasibility of a play-based telehealth intervention program for children with Prader-Willi syndrome. J Autism Dev Disord. 47(9):2814-2825. doi: 10.1007/s10803-017-3196-z.

Dorsey, E. R. and Topol, E. J., M.D. (2016). State of Telehealth. New England Journal of Medicine, 375(14), 1399-1400. doi:10.1056/nejmc1610233

Douthit, N., Kiv, S., Dwolatzky, T., and Biswas, S. (2015). Exposing some important barriers to healthcare access in the rural USA. Public Health. 129:6, 611-620. doi:10.1016/j.puhe.2015.04.001

Eckert, M., Volmerg, J. S., and Friedrich, C. M. (2019). Augmented reality in medicine: systematic and bibliographic review. JMIR mHealth and uHealth, 7(4), e10967. doi:10.2196/10967 
Eslami Jahromi M, and Ahmadian L. (2018). Evaluating satisfaction of patients with stutter regarding the tele-speech therapy method and infrastructure. Int J Med Inform. 115:128-133. doi: 10.1016/j.ijmedinf.2018.03.004.

Fermin, J. L., and Tan, M. J. T. (2020). The Need for the Establishment of Biomedical Engineering as an Academic and Professional Discipline in the Philippines-A Quantitative Argument. IEEE Access. doi:10.1109/ACCESS.2020.3046655

Fernandez-Marcelo, P. G., Ho, B. L., Faustorilla Jr, J. F., Evangelista, A. L., Pedrena, M., and Marcelo, A. (2012). Emerging eHealth directions in the Philippines. Yearbook of medical informatics, 21(01), 144-152. doi: 10.1055/S-0038-1639446

Geneva: World Health Organizations (2011). mHealth: New Horizons for Health Through Mobile Technologies.https://www.who.int/goe/publications/goe_mhealth_web.pdf [Accessed July 12, 2021]

Geneva: World Health Organization (2021). The 2021 update: Global health workforce statistics. https://www.who.int/data/gho/data/themes/topics/indicator-groups/indicator-group-details/GHO /medical-doctors [Accessed July 1, 2021].

Holderried, M., Hoeper A., and Holderried, F. et al. (2021). Attitude and potential benefits of modern information and communication technology use and telemedicine in cross-sectoral solid organ transplant care. Scientific Reports 11, 9037. doi: 10.1038/s41598-021-88447-6

House of Representatives (2018). House bill no. 7153.

https://congress.gov.ph/legisdocs/basic_17/HB07153.pdf [Accessed July 1, 2021].

House of Representatives (2020). House bill no. 7422.

https://www.congress.gov.ph/legisdocs/basic_18/HBo7422.pdf [Accessed July 1, 2021].

Intan Sabrina, M., and Defi, I. R. (2021). Telemedicine guidelines in south East Asia-A Scoping Review. Frontiers in Neurology, 11, 1760. doi: 10.3389/fneur.2020.581649

Intawong, K., Olson, D., and Chariyalertsak, S. (2021). Application technology to fight the COVID-19 pandemic: Lessons learned in Thailand. Biochemical and biophysical research communications, 534, 830-836. doi: 10.1016/j.bbrc.2020.10.097

Jagadeeswari, V., Subramaniyaswamy, V., Logesh, R., and Vijayakumar, V. (2018). A study on medical Internet of Things and Big Data in personalized healthcare system. Health information science and systems, 6(1), 1-20. doi:10.1007/s13755-018-0049-X

Jalal, S., Nicolaou, S., and Parker, W. (2019). Artificial intelligence, radiology, and the way forward. Canadian association of radiologists journal, 7o(1), 10-12. doi:10.1016/j.carj.2018.09.004 
Kanchanachitra, C., Lindelow, M., Johnston, T., Hanvoravongchai, P., Lorenzo, F. M., and Huong, N. L., et al. (2011). Human resources for health in southeast Asia: Shortages, distributional challenges, and international trade in health services. The Lancet. 377:9767, 769-781.

doi:10.1016/So140-6736(10)62035-1

Koonin, L. M., Hoots, B., Tsang, C. A., Leroy, Z., Farris, K., and Jolly, B., et al. (2020). Trends in the use of telehealth during the emergence of the COVID-19 pandemic-United States, January-March 2020. Morbidity and Mortality Weekly Report, 69(43), 1595. doi:10.15585/mmwr.mm6943a3

Kruse, C. S., Krowski, N., Rodriguez, B., Tran, L., Vela, J., and Brooks, M. (2017). Telehealth and patient satisfaction: a systematic review and narrative analysis. BMJ open, 7(8), e016242.

doi:10.1136/bmjopen-2017-016242

Lim, J., Chan, M. M. H., Alsahoff, F., Z. and Ha, D. (2014). Innovations in non-communicable diseases management in ASEAN: A case series. Global Health Action. 7:1. doi:10.3402/gha.v7.25110

Ling, M. L., Apisarnthanarak, A., and Madriaga, G. (2015). The Burden of healthcare-associated infections in Southeast Asia: A systematic literature review and meta-analysis, Clinical Infectious Diseases, 60:11, 1690-1699. doi:10.1093/cid/civo95

Malasinghe, L. P., Ramzan, N., and Dahal, K. (2019). Remote patient monitoring: a comprehensive study. Journal of Ambient Intelligence and Humanized Computing, 10(1), 57-76.

doi:10.1007/s12652-017-0598-x

Merriman, E., Corwin, P., and Ikram R. (2002). Toys are a potential source of cross-infection in general practitioners' waiting rooms. The British Journal of General Practice. 52:475, 138-140.

Michtalik, H. J., Yeh, H. C., Pronovost, P. J., and Brotman, D. J. (2013). Impact of attending physician workload on patient care: A survey of hospitalists. JAMA Internal Medicine. 173:5, 375-377. doi:10.1001/jamainternmed.2013.1864

Monaghesh, E., and Hajizadeh, A. (2020). The role of telehealth during COVID-19 outbreak: a systematic review based on current evidence. BMC Public Health, 2O(1).

doi:10.1186/s12889-020-09301-4

Moser, D. K., Kimble, L. P., Alberts, M. J., Alonzo, A., Croft, J. B, and Dracup, K., et al. (2006). Reducing delay in seeking treatment by patients with acute coronary syndrome and stroke: A scientific statement from the American Heart Association Council on Cardiovascular Nursing and Stroke Council. Circulation. 114, 168-182. doi:10.1161/circulationaha.106.176040

Nair, M. and Webster, P. (2013). Health professionals' migration in emerging market economies: patterns, causes and possible solutions. Journal of Public Health. 35:1, 157-163. doi:10.1093/pubmed/fdso87

Nit, B., Kobashi, Y., Vory, S., Lim, S., Chea, S., Ito, S., and Tsubokura, M. (2021). The introduction of telemedicine is required immediately in Cambodia: Barriers and lessons from COVID-19. Journal of global health, 11, 03047. doi:10.7189/jogh.11.03047 
Nguyen, Q. T., Naguib, R. N. G., Ghani, M. K. A., Bali, R. K., Marshall, I. M., and Phuong, et al. (2008). An analysis of the healthcare informatics and systems in Southeast Asia: a current perspective from seven countries. International Journal of Electronic Healthcare, 4(2), 184. doi:10.1504/ijeh.2008.019792

Nochomovitz, M. and Sharma, R. (2017). Is it time for a new medical specialty? The medical virtualist. Journal of the American Medical Association. doi:10.1001/jama.2017.17094

Organisation for Economic Co-operation and Development (2021), Economic Outlook for Southeast Asia, China and India 2021: Reallocating Resources for Digitalisation, OECD Publishing, Paris, doi10.1787/711629f8-en.

Philippine E-Health and Telemedicine Development Act, H.R. 7422, 18th Cong. (2020). https://www.congress.gov.ph/legisdocs/basic_18/HBo7422.pdf [Accessed July 12, 2021]

Philippine Statistics Authority (2020). How much is the Vulnerable and Elderly Population Aged 60 and Above Spending for Health Care?. Philippine Statistics Authority.

https://psa.gov.ph/pnha-press-release/node/162928 [Accessed July 12, 2021]

Philippine Statistics Authority (2020). Health Spending Grew by 10.9 Percent in 2019. Philippine Statistics Authority. https://psa.gov.ph/pnha-press-release/node/163258 [Accessed July 12, 2021]

Raghavan, A., Demircioglu, M. A., and Taeihagh, A. (2021). Public Health Innovation through Cloud Adoption: A Comparative Analysis of Drivers and Barriers in Japan, South Korea, and Singapore. International Journal of Environmental Research and Public Health, 18(1), 334.

doi:10.339o/ijerph18010334

Raghupathi, V., and Raghupathi, W. (2020). Healthcare Expenditure and Economic Performance: Insights From the United States Data. Frontiers in public health, 8, 156.

doi:10.3389/fpubh.2020.00156

Salac, R. A., and Kim, Y. S. (2016). A Study on The Internet Connectivity in The Philippines. Asia Pacific Journal of Business Review, 1(1), 67-88. doi:10.20522/apjbr.2016.1.1.67

Singapore Ministry of Health. (2021). Listing of direct telemedicine providers: transition approach prior to licensing under the Healthcare Services Act (HCSA).

https://www.moh.gov.sg/licensing-and-regulation/telemedicine [Accessed July 12, 2021]

Siriwardhana, Y., Gür, G., Ylianttila, M., and Liyanage, M. (2020). The role of $5 \mathrm{G}$ for digital healthcare against COVID-19 pandemic: Opportunities and challenges. ICT Express. doi:10.1016/j.icte.2020.10.002

Somsiri, V., Asdornwised, U., O'Connor, M., Suwanugsorn, S., and Chansatitporn, N. (2020). Effects of a Transitional Telehealth Program on Functional Status, Rehospitalization, and Satisfaction With Care in Thai Patients with Heart Failure. Home Health Care Management \& Practice, 33(2), 72-80. doi: $10.1177 / 1084822320969400$ 
Swanepoel, D. W., Clark, J. L., Koekemoer, D., Hall Iii, J. W., Krumm, M., and Ferrari, D. V., et al. (2010). Telehealth in audiology: The need and potential to reach underserved communities.

International Journal of Audiology, 49(3), 195-202. doi:10.3109/14992020903470783

Taber, J. M., Leyva, B., and Persoskie, A. (2015). Why do people avoid medical care? A qualitative study using national data. Journal of General Internal Medicine. 30:3, 290-297.

doi:10.1007/s11606-014-3089-1

Tareq, M. S., Rahman, T., Hossain, M., and Dorrington, P. (2021). Additive manufacturing and the COVID-19 challenges: An in-depth study. Journal of Manufacturing Systems.

doi:10.1016/j.jmsy.2020.12.021

Thailand Ministry of Public Health. (2017). eHealth Strategy (2017-2026).

https://ehealth.moph.go.th/index.php/resources/draft-ehealth-strategy-ministry-of-public-health-2 016-2021 [Accessed July 12, 2021]

Ting, D. S., Lin, H., Ruamviboonsuk, P., Wong, T. Y., and Sim, D. A. (2020). Artificial intelligence, the internet of things, and virtual clinics: ophthalmology at the digital translation forefront. The Lancet Digital Health, 2(1), e8-e9. doi:10.1016/S2589-7500(19)30217-1

Van Minh, H., Pocock, N. S., Chaiyakunapruk, N., Chhorvann, C., Duc, H. A., and Hanvoravongchai, P., et al. (2014). Progress toward universal health coverage in ASEAN. Global Health Action. 7:1. doi:10.3402/gha.v7.25856

Wade, V.A., Karnon, J., Elshaug, A.G., and Hiller, J.E. (2010). A systematic review of economic analyses of telehealth services using real time video communication. BMC Health Serv Res 10, 233. doi:10.1186/1472-6963-10-233

Weber, E., Miller, S. J., Astha, V., Janevic, T., and Benn, E. (2020). Characteristics of telehealth users in NYC for COVID-related care during the coronavirus pandemic. Journal of the American Medical Informatics Association, 27(12), 1949-1954. doi:10.1093/jamia/ocaa216

World Health Organizations (2020). Implementing telemedicine services during COVID-19 : guiding principles and considerations for a stepwise approach.

http://iris.wpro.who.int/handle/10665.1/14651 [Accessed July 12, 2021]

Xu, T., Pujara, S., Sutton, S., and Rhee, M. (2018). Telemedicine in the Management of Type 1 Diabetes. Prev Chronic Dis. 15:170168. doi:10.5888/pcd15.170168

Ye, J. (2020). The role of health technology and informatics in a global public health emergency: practices and implications from the COVID-19 pandemic. JMIR medical informatics, 8(7), e19866. doi:10.2196/19866

Zhou, X., Snoswell, C. L., Harding, L. E., Bambling, M., Edirippulige, S., Bai, X., and Smith, A. C. (2020). The role of telehealth in reducing the mental health burden from COVID-19. Telemedicine and e-Health, 26(4), 377-379. doi:10.1089/tmj.2020.0068 